\title{
FURTHER RESULTS ON APPROXIMATION BY DOUBLE SINGULAR INTEGRAL OPERATORS WITH RADIAL KERNELS
}

\section{GUMRAH UYSAL and ERTAN IBIKLI}

Department of Mathematics

Faculty of Science

Karabuk University

Karabuk

Turkey

e-mail: guysal@karabuk.edu.tr

Department of Mathematics

Faculty of Science

Ankara University

Anadolu, Ankara

Turkey

e-mail: Ertan.Ibikli@ankara.edu.tr

\begin{abstract}
This paper contains some results on the point-wise convergence of a family of singular integral operators with radial kernels given in the following form:

$$
L_{\lambda}(f ; x, y)=\iint_{\mathbb{R}^{2}} f(t, s) H_{\lambda}(t-x, s-y) d t d s, \quad(x, y) \in \mathbb{R}^{2}, \quad \lambda \in \Lambda,
$$

2010 Mathematics Subject Classification: Primary 41A35; Secondary 41A25, 45P05, 47A58,

Keywords and phrases: generalized Lebesgue point, radial kernel, point-wise convergence,

Received October 25, 2015
\end{abstract} $47 \mathrm{~B} 65$. rate of convergence.

(C) 2015 Scientific Advances Publishers 
where $\Lambda$ is a set of non-negative numbers with accumulation point $\lambda_{0}$. Here,

the functions $f: \mathbb{R}^{2} \rightarrow \mathbb{R}$ are measurable in the sense of Lebesgue.

\section{Introduction}

Approximation with linear integral operators or suitable functions finds frequent application in many areas of mathematics including Fourier analysis. The following integral operator is a familiar example:

$$
L_{n}(f ; x)=\int_{-\pi}^{\pi} f(t) K_{n}(t, x) d t, \quad x \in(-\pi, \pi), n \in \mathbb{N}
$$

where $K_{n}: \mathbb{R} \times \mathbb{R} \rightarrow \mathbb{R}_{0}^{+}$denotes a family of periodic kernels satisfying usual conditions for approximate identities.

In [16], Taberski studied the point-wise approximation of periodic and integrable functions on $\langle-\pi, \pi\rangle$, where $\langle-\pi, \pi\rangle$ is an arbitrary closed, semi-closed or open interval. The work used a two parameter family of singular integral operators of the form:

$$
L_{\lambda}(f ; x)=\int_{-\pi}^{\pi} f(t) K_{\lambda}(t-x) d t, \quad x \in\langle-\pi, \pi\rangle, \lambda \in \Lambda,
$$

where $K_{\lambda}: \mathbb{R} \rightarrow \mathbb{R}_{0}^{+}$denotes a family of periodic kernels satisfying suitable conditions and $\Lambda$ is a given set of non-negative numbers with accumulation point $\lambda_{0}$. Following Taberki's line, [4], [9], and [6] obtained more general results. As concerns the study of integral operators in several settings, the reader may see also, e.g., [1, 2] and [13]-[22].

Taberski [14] advanced his analysis to double singular integral operators depending on three parameters of the form:

$$
L_{\lambda}(f ; x, y)=\iint_{Q} f(t, s) K_{\lambda}(t-x, s-y) d t d s, \quad(x, y) \in Q,
$$


where $Q=\langle-\pi, \pi\rangle \times\langle-\pi, \pi\rangle$ is an arbitrary closed, semi-closed or open region and $K_{\lambda}: \mathbb{R}^{2} \rightarrow \mathbb{R}_{0}^{+}$stands for a family of kernels satisfying some conditions. Those results were later used by Siudut $[11,12]$ presenting considerable theorems. Then, Rydzewska [8] improved the results of [15]. In recent papers [18]-[20], the kernel function was chosen as a radial function and the domain of integration was replaced by an arbitrary region $\langle a, b\rangle \times\langle c, d\rangle$. In the light of these modifications, some point-wise approximation theorems are presented. Also, we shall mention the papers [7] and [15] which are important works on weighted approximation by singular integral operators. Those contain detailed information regarding the characterization of the weight functions with examples.

The current manuscript deals with point-wise convergence of a family of singular integral operators with radial kernels given in the following form:

$$
L_{\lambda}(f ; x, y)=\iint_{\mathbb{R}^{2}} f(t, s) H_{\lambda}(t-x, s-y) d t d s, \quad(x, y) \in \mathbb{R}^{2}, \lambda \in \Lambda,
$$

where $\Lambda$ is a set of non-negative numbers with accumulation point $\lambda_{0}$ at a $p$-generalized Lebesgue point of $f \in L_{p, \varphi}\left(\mathbb{R}^{2}\right)$. Here, $L_{p, \varphi}\left(\mathbb{R}^{2}\right)$ is the collection of all measurable functions for which $\left|\frac{f}{\varphi}\right|^{p}$ is integrable on $\mathbb{R}^{2}(1 \leq p<\infty)$ provided $\varphi: \mathbb{R}^{2} \rightarrow \mathbb{R}^{+}$is a weight function, which is measurable and bounded on any bounded subset of $\mathbb{R}^{2}$.

\section{Preliminaries}

In this section, we introduce the main definitions which have vital roles in this work.

Definition 1. A function $H \in L_{1}\left(\mathbb{R}^{2}\right)$, is said to be radial, if there exists a function $K: \mathbb{R}_{0}^{+} \rightarrow \mathbb{R}$ such that $H(t, s)=K\left(\sqrt{t^{2}+s^{2}}\right)$ almost everywhere [3]. 
Now, we give another characterization of $p$-generalized Lebesgue point for the functions of two variables.

Definition 2. A $p$-generalized Lebesgue point of a locally (or globally) integrable function $g: \mathbb{R}^{2} \rightarrow \mathbb{R}$ is a point $\left(x_{0}, y_{0}\right) \in \mathbb{R}^{2}$ satisfying

$$
\begin{gathered}
\lim _{(h, k) \rightarrow(0,0)}\left(\frac{1}{h^{\alpha+1} k^{\beta+1}} \int_{0}^{h} \int_{0}^{k}\left|g\left(t+x_{0}, s+y_{0}\right)-g\left(x_{0}, y_{0}\right)\right|^{p} d t d s\right)^{\frac{1}{p}}=0, \\
1 \leq p<\infty, 0 \leq \alpha, \beta<1 .
\end{gathered}
$$

If one takes $\alpha=\beta$, then the above definition reduces to definition of $p$-generalized Lebesgue point given in [20]. For some examples concerning this point, see [18].

Definition 3 (Class $A_{\varphi}$ ). Let $H_{\lambda}: \mathbb{R}^{2} \rightarrow \mathbb{R}_{0}^{+}$be a radial function, i.e., there exists a function $K_{\lambda}: \mathbb{R}_{0}^{+} \rightarrow \mathbb{R}_{0}^{+}$such that $H_{\lambda}(t, s):=K_{\lambda}$ $\left(\sqrt{t^{2}+s^{2}}\right)$ holds almost everywhere on $\mathbb{R}^{2}$ for each fixed $\lambda \in \Lambda$. Further, let $H_{\lambda}: \mathbb{R}^{2} \rightarrow \mathbb{R}_{0}^{+}$be a family of radial kernels which are integrable on $\mathbb{R}^{2}$, for each fixed $\lambda \in \Lambda$ and the weight function $\varphi: \mathbb{R}^{2} \rightarrow \mathbb{R}^{+}$is bounded and measurable on any bounded subset of $\mathbb{R}^{2}$ and satisfies the following inequality:

$$
\varphi(u+t, v+s) \leq \varphi(u, v) \varphi(t, s), \quad(t, s) \in \mathbb{R}^{2}, \quad(u, v) \in \mathbb{R}^{2} .
$$

We say that $H_{\lambda}(t, s)$ belongs to class $A_{\varphi}$, if the following conditions are satisfied:

(a) At a $p$-generalized Lebesgue point $\left(x_{0}, y_{0}\right) \in \mathbb{R}^{2}$ of the weight function $\varphi$, the following relation:

$$
\lim _{(x, y, \lambda) \rightarrow\left(x_{0}, y_{0}, \lambda_{0}\right)} \frac{1}{\varphi\left(x_{0}, y_{0}\right)} \iint_{\mathbb{R}^{2}} \varphi(t, s) K_{\lambda}\left(\sqrt{(t-x)^{2}+(s-y)^{2}}\right) d t d s=1
$$

holds. 
(b) $\forall \xi>0$,

$$
\lim _{(x, y, \lambda) \rightarrow\left(x_{0}, y_{0}, \lambda_{0}\right)} \sup _{\xi \leq \sqrt{\left(t^{2}+s^{2}\right)}}\left[\varphi(t, s) K_{\lambda}\left(\sqrt{(t-x)^{2}+(s-y)^{2}}\right)\right]=0 .
$$

(c) $\forall \xi>0$,

$$
\lim _{(x, y, \lambda) \rightarrow\left(x_{0}, y_{0}, \lambda_{0}\right)}\left[\int_{\xi \leq \sqrt{\left(t^{2}+s^{2}\right)}} \varphi(t, s) K_{\lambda}\left(\sqrt{(t-x)^{2}+(s-y)^{2}}\right) d t d s\right]=0 .
$$

(d) $K_{\lambda}\left(\sqrt{t^{2}+s^{2}}\right)$ is monotonically increasing with respect to $t$ on $(-\infty, 0]$ and similarly, $K_{\lambda}\left(\sqrt{t^{2}+s^{2}}\right)$ is monotonically increasing with respect to $s$ on $(-\infty, 0]$ for any $\lambda \in \Lambda$. Analogously, $K_{\lambda}\left(\sqrt{t^{2}+s^{2}}\right)$ is bimonotonically increasing with respect to $(t, s)$ on $[0, \infty) \times[0, \infty)$ and $(-\infty, 0] \times(-\infty, 0]$ and bimonotonically decreasing with respect to $(t, s)$ on $[0, \infty) \times(-\infty, 0]$ and $(-\infty, 0] \times[0, \infty)$ for any $\lambda \in \Lambda$.

(e) $\left\|\varphi K_{\lambda}\right\|_{L_{1}\left(\mathbb{R}^{2}\right)} \leq M<\infty$, for all $\lambda \in \Lambda$.

(f) For fixed $\left(t_{0}, s_{0}\right) \in \mathbb{R}^{2}, K_{\lambda}\left(\sqrt{t_{0}^{2}+s_{0}^{2}}\right)$ tends to infinity as $\lambda$ tends to $\lambda_{0}$.

Note that, throughout this paper, we suppose that the kernel $H_{\lambda}(t, s)$ belongs to class $A_{\varphi}$.

Remark 1. If the function $g: \mathbb{R}^{2} \rightarrow \mathbb{R}$ is bimonotonic on $\left[\alpha_{1}, \alpha_{2}\right] \times$ $\left[\beta_{1}, \beta_{2}\right] \subset \mathbb{R}^{2}$, then the equality given by

$$
\begin{aligned}
V\left(g ;\left[\alpha_{1}, \alpha_{2}\right] \times\left[\beta_{1}, \beta_{2}\right]\right) & =\bigvee_{\alpha_{1}}^{\alpha_{2}} \bigvee_{1}^{\beta_{2}}(g(t, s)) \\
& =\left|g\left(\alpha_{1}, \beta_{1}\right)-g\left(\alpha_{1}, \beta_{2}\right)-g\left(\alpha_{2}, \beta_{1}\right)+g\left(\alpha_{2}, \beta_{2}\right)\right|
\end{aligned}
$$

holds $[14,5]$. 


\section{Existence of the Operator}

The following lemma gives the existence of the operators defined by (4). For this kind of existence theorems, see [21, 20].

Lemma 1. If $f \in L_{p, \varphi}\left(\mathbb{R}^{2}\right)$, then the operators $L_{\lambda}(f ; x, y)$ define a continuous transformation acting on $L_{p, \varphi}\left(\mathbb{R}^{2}\right)$.

Proof. Since $L_{\lambda}(f ; x, y)$ is linear, it is sufficient to show that the norm given in the form

$$
\left\|L_{\lambda}\right\|_{\varphi}=\sup _{f \neq 0} \frac{\left\|L_{\lambda}(f ; x, y)\right\|_{L_{p, \varphi}\left(\mathbb{R}^{2}\right)}}{\|f\|_{L_{p, \varphi}\left(\mathbb{R}^{2}\right)}}
$$

is bounded. Here the norm $\|f\|_{L_{p, \varphi}\left(\mathbb{R}^{2}\right)}$ given by

$$
\|f\|_{L_{p, \varphi}\left(\mathbb{R}^{2}\right)}=\left(\iint_{\mathbb{R}^{2}}\left|\frac{f(t, s)}{\varphi(t, s)}\right|^{p} d t d s\right)^{\frac{1}{p}}, \quad 1 \leq p<\infty .
$$

See, for example, $[7,15]$.

Now, let $1<p<\infty$. In view of (5) and generalized Minkowski inequality [13], we have

$$
\begin{aligned}
\left\|L_{\lambda}(f ; x, y)\right\|_{L_{1, \varphi}\left(\mathbb{R}^{2}\right)} & \\
= & \left.\left.\iiint_{\mathbb{R}^{2}}\left|\frac{1}{\varphi(x, y)}\right| \iint_{\mathbb{R}^{2}} f(t, s) K_{\lambda}\left(\sqrt{(t-x)^{2}+(s-y)^{2}}\right) d t d s \mid\right)^{p} d x d y\right)^{\frac{1}{p}} \\
& \leq \iint_{\mathbb{R}^{2}} K_{\lambda}\left(\sqrt{t^{2}+s^{2}}\right) \varphi(t, s)\left(\iint_{\mathbb{R}^{2}}\left|\frac{f(t+x, s+y)}{\varphi(t+x, s+y)}\right|^{p} d x d y\right)^{\frac{1}{p}} d t d s \\
& =\left\|\varphi K_{\lambda}\right\|_{L_{1}\left(\mathbb{R}^{2}\right)} \leq M\|f\|_{L_{p, \varphi}\left(\mathbb{R}^{2}\right)}
\end{aligned}
$$


Thus, the proof is completed for $1<p<\infty$. One may easily prove the assertion for the case $p=1$ by using similar method. Thus, this case is omitted. Hence the proof is finished.

\section{Point-wise Convergence}

The following theorem gives a point-wise convergence of the integral operators of type (4) at a common $p$-generalized Lebesgue point of $f \in L_{p, \varphi}\left(\mathbb{R}^{2}\right)$ and the weight function $\varphi: \mathbb{R}^{2} \rightarrow \mathbb{R}^{+}$. It should be noticed that local integrability of $\varphi: \mathbb{R}^{2} \rightarrow \mathbb{R}^{+}$is guaranteed by the properties of it.

Theorem 1. If $\left(x_{0}, y_{0}\right) \in \mathbb{R}^{2}$ is a common p-generalized Lebesgue point of $f \in L_{p, \varphi}\left(\mathbb{R}^{2}\right)$ and $\varphi$, then

$$
\lim _{(x, y, \lambda) \rightarrow\left(x_{0}, y_{0}, \lambda_{0}\right)} L_{\lambda}(f ; x, y)=f\left(x_{0}, y_{0}\right),
$$

on any set $Z$ on which the function

$$
\begin{aligned}
& \sup _{(t, s) \in B_{\delta}} \varphi(t, s)\left\{\int_{x_{0}-\delta}^{x_{0}+\delta} \int_{y_{0}-\delta}^{y_{0}+\delta} K_{\lambda}\left(\sqrt{(t-x)^{2}+(s-y)^{2}}\right)\left|\left\{\left|x_{0}-t\right|^{\alpha+1}\right\}_{t}^{\prime}\right|\right. \\
& \quad \times\left|\left\{\left|y_{0}-s\right|^{\beta+1}\right\}_{s}^{\prime}\right| d t d s+2\left|y_{0}-y\right|^{\beta+1} \int_{x_{0}-\delta}^{x_{0}+\delta} K_{\lambda}(|t-x|)\left|\left\{\left|x_{0}-t\right|^{\alpha+1}\right\}_{t}^{\prime}\right| d t \\
& +2\left|x_{0}-x\right|^{\alpha+1} \int_{y_{0}-\delta}^{y_{0}+\delta} K_{\lambda}(|s-y|)\left|\left\{\left|y_{0}-s\right|^{\beta+1}\right\}_{s}^{\prime}\right| d s \\
& \left.+4 K_{\lambda}(0)\left|x_{0}-x\right|^{\alpha+1}\left|y_{0}-y\right|^{\beta+1}\right\}
\end{aligned}
$$

where $B_{\delta}:=\left\{(t, s):\left(t-x_{0}\right)^{2}+\left(s-y_{0}\right)^{2}<\delta^{2},\left(x_{0}, y_{0}\right) \in \mathbb{R}^{2}\right\}$, is bounded as $(x, y, \lambda)$ tends to $\left(x_{0}, y_{0}, \lambda_{0}\right)$. 
Proof. Suppose that $\left(x_{0}, y_{0}\right) \in \mathbb{R}^{2}$ is a $p$-generalized Lebesgue point of function $f \in L_{p, \varphi}\left(\mathbb{R}^{2}\right)$. Besides, we may assume that $0<x_{0}-x<\delta / 2$ and $0<y_{0}-y<\delta / 2$ for $0<\delta<\infty$. Therefore, for all given $\varepsilon>0$, there exists a $\delta>0$ such that for all $h$ and $k$ satisfying $0<h, k \leq \delta$, we have the inequality:

$$
\int_{x_{0}}^{x_{0}+h} \int_{y_{0}-k}^{y_{0}}\left|\frac{f(t, s)}{\varphi(t, s)}-\frac{f\left(x_{0}, y_{0}\right)}{\varphi\left(x_{0}, y_{0}\right)}\right|^{p} d t d s<\varepsilon^{p} h^{\alpha+1} k^{\beta+1} .
$$

In view of condition (a), we have

$$
\begin{aligned}
\left|L_{\lambda}(f ; x, y)-f\left(x_{0}, y_{0}\right)\right| & \\
\leq & \iint_{\mathbb{R}^{2}}\left|\frac{f(t, s)}{\varphi(t, s)}-\frac{f\left(x_{0}, y_{0}\right)}{\varphi\left(x_{0}, y_{0}\right)}\right| \varphi(t, s) K_{\lambda}\left(\sqrt{(t-x)^{2}+(s-y)^{2}}\right) d t d s \\
& +\left|\frac{f\left(x_{0}, y_{0}\right)}{\varphi\left(x_{0}, y_{0}\right)}\right|\left|\iint_{\mathbb{R}^{2}} \varphi(t, s) K_{\lambda}\left(\sqrt{(t-x)^{2}+(s-y)^{2}}\right) d t d s-\varphi\left(x_{0}, y_{0}\right)\right| \\
= & I_{1}+I_{2} .
\end{aligned}
$$

Let $1<p<\infty$. Using Hölder's inequality, see [10], for the term $I_{1}$, we have

$$
\begin{aligned}
\mid L_{\lambda}(f ; x, y) & -f\left(x_{0}, y_{0}\right) \mid \\
\leq & \left(\iint_{\mathbb{R}^{2}}\left|\frac{f(t, s)}{\varphi(t, s)}-\frac{f\left(x_{0}, y_{0}\right)}{\varphi\left(x_{0}, y_{0}\right)}\right|^{p} \varphi(t, s) K_{\lambda}\left(\sqrt{(t-x)^{2}+(s-y)^{2}}\right) d t d s\right)^{\frac{1}{p}} \\
& \times\left(\iint_{\mathbb{R}^{2}} \varphi(t, s) K_{\lambda}\left(\sqrt{(t-x)^{2}+(s-y)^{2}}\right) d t d s\right)^{\frac{1}{q}}+I_{2} .
\end{aligned}
$$


Since whenever for $m, n$ being positive numbers the inequality $(m+n)^{p} \leq 2^{p}\left(m^{p}+n^{p}\right)$ holds, see [10], by taking $p$-th power of both sides, we have

$$
\begin{aligned}
& \left|L_{\lambda}(f ; x, y)-f\left(x_{0}, y_{0}\right)\right|^{p} \\
& \leq 2^{p} \iint_{\mathbb{R}^{2}}\left|\frac{f(t, s)}{\varphi(t, s)}-\frac{f\left(x_{0}, y_{0}\right)}{\varphi\left(x_{0}, y_{0}\right)}\right|^{p} \varphi(t, s) K_{\lambda}\left(\sqrt{(t-x)^{2}+(s-y)^{2}}\right) d t d s \\
& \quad \times\left(\iint_{\mathbb{R}^{2}} \varphi(t, s) K_{\lambda}\left(\sqrt{(t-x)^{2}+(s-y)^{2}}\right) d t d s\right)^{p / q} \\
& \quad+2^{p}\left|\frac{f\left(x_{0}, y_{0}\right)}{\varphi\left(x_{0}, y_{0}\right)}\right|^{p}\left|\iint_{\mathbb{R}^{2}} \varphi(t, s) K_{\lambda}\left(\sqrt{(t-x)^{2}+(s-y)^{2}}\right) d t d s-\varphi\left(x_{0}, y_{0}\right)\right|^{p} \\
& =2^{p} I_{11} I^{*}+2^{p} I_{21} .
\end{aligned}
$$

In view of condition (a) of class $A_{\varphi}, I_{21} \rightarrow 0$ and $I^{*} \rightarrow \varphi\left(x_{0}, y_{0}\right)^{p / q}<\infty$ as $(x, y, \lambda)$ tends to $\left(x_{0}, y_{0}, \lambda_{0}\right)$, respectively. It is easy to see that $I_{11}$ can be written in the form:

$$
\begin{aligned}
I_{11} & =\left\{\iint_{\mathbb{R}^{2} \backslash B_{\delta}}+\iint_{B_{\delta}}\right\}\left|\frac{f(t, s)}{\varphi(t, s)}-\frac{f\left(x_{0}, y_{0}\right)}{\varphi\left(x_{0}, y_{0}\right)}\right|^{p} \varphi(t, s) K_{\lambda}\left(\sqrt{(t-x)^{2}+(s-y)^{2}}\right) d t d s \\
& =I_{111}+I_{112},
\end{aligned}
$$

where $B_{\delta}:=\left\{(t, s):\left(t-x_{0}\right)^{2}+\left(s-y_{0}\right)^{2}<\delta^{2},\left(x_{0}, y_{0}\right) \in \mathbb{R}^{2}\right\}$. Since 


$$
\begin{aligned}
I_{111} 2^{-p} & \leq \sup _{(t, s) \in \mathbb{R}^{2} \backslash B_{\delta}}\left[\varphi(t, s) K_{\lambda}\left(\sqrt{(t-x)^{2}+(s-y)^{2}}\right)\right]\|f\|_{L_{p, \varphi}}^{p}\left(\mathbb{R}^{2}\right) \\
& +\left|\frac{f\left(x_{0}, y_{0}\right)}{\varphi\left(x_{0}, y_{0}\right)}\right|^{p} \iint_{\mathbb{R}^{2} \backslash B_{\delta}} \varphi(t, s) K_{\lambda}\left(\sqrt{(t-x)^{2}+(s-y)^{2}}\right) d t d s
\end{aligned}
$$

holds, by condition (b) and (c) of class $A_{\varphi}, I_{111} \rightarrow 0$ as $(x, y, \lambda) \rightarrow$ $\left(x_{0}, y_{0}, \lambda_{0}\right)$.

Let us prove that $I_{112}$ tends to zero as $(x, y, \lambda)$ tends to $\left(x_{0}, y_{0}, \lambda_{0}\right)$. Since $\varphi(t, s)$ is bounded on arbitrary bounded subsets of $\mathbb{R}^{2}$, it is bounded on $B_{\delta}$. Therefore, it is easy to see that the following inequality holds for $I_{112}$ :

$$
\begin{aligned}
I_{112} & <\sup _{(t, s) \in B_{\delta}} \varphi(t, s) \int_{x_{0}-\delta}^{x_{0}+\delta} \int_{y_{0}-\delta}^{y_{0}+\delta}\left|\frac{f(t, s)}{\varphi(t, s)}-\frac{f\left(x_{0}, y_{0}\right)}{\varphi\left(x_{0}, y_{0}\right)}\right|^{p} K_{\lambda}\left(\sqrt{(t-x)^{2}+(s-y)^{2}}\right) d t d s \\
& =\sup _{(t, s) \in B_{\delta}} \varphi(t, s) I_{112}^{*} .
\end{aligned}
$$

Splitting the integral $I_{112}^{*}$ into four parts, we have

$$
\begin{aligned}
I_{112}^{*} \leq & \left\{\int_{x_{0}}^{x_{0}+\delta} \int_{y_{0}-\delta}^{y_{0}}+\int_{x_{0}-\delta}^{x_{0}} \int_{y_{0}-\delta}^{y_{0}}\right\}\left|\frac{f(t, s)}{\varphi(t, s)}-\frac{f\left(x_{0}, y_{0}\right)}{\varphi\left(x_{0}, y_{0}\right)}\right|^{p} K_{\lambda}\left(\sqrt{(t-x)^{2}+(s-y)^{2}}\right) d t d s \\
& +\left\{\int_{x_{0}-\delta}^{x_{0}} \int_{y_{0}}^{y_{0}+\delta}+\int_{x_{0}}^{x_{0}+\delta} \int_{y_{0}}^{y_{0}+\delta}\right\}\left|\frac{f(t, s)}{\varphi(t, s)}-\frac{f\left(x_{0}, y_{0}\right)}{\varphi\left(x_{0}, y_{0}\right)}\right|^{p} \\
& \times K_{\lambda}\left(\sqrt{(t-x)^{2}+(s-y)^{2}}\right) d t d s \\
= & I_{1121}^{*}+I_{1122}^{*}+I_{1123}^{*}+I_{1124}^{*} .
\end{aligned}
$$

Let us consider the integral $I_{1121}^{*}$. 
We define the variations:

$$
\begin{aligned}
& E_{1}(u, v):=\left\{\begin{array}{cc}
\bigvee_{u}^{x_{0}+\delta-x} \bigvee_{y_{0}-\delta-y}^{v}\left(K_{\lambda}\left(\sqrt{t^{2}+s^{2}}\right)\right), & x_{0}-x \leq u<x_{0}+\delta-x, \\
& y_{0}-\delta-y<v \leq y_{0}-y, \\
0, & \text { otherwise. }
\end{array}\right. \\
& E_{2}(u):=\left\{\begin{array}{cl}
\bigvee_{u}^{x_{0}+\delta-x}\left(K_{\lambda}\left(\sqrt{t^{2}+\left(y_{0}-\delta-y\right)^{2}}\right)\right), & x_{0}-x \leq u<x_{0}+\delta-x \\
0, & \text { otherwise }
\end{array}\right. \\
& E_{3}(v):=\left\{\begin{array}{cl}
\bigvee_{y_{0}-\delta-y}^{v}\left(K_{\lambda}\left(\sqrt{\left(x_{0}-x+\delta\right)^{2}+s^{2}}\right)\right), & y_{0}-\delta-y<v \leq y_{0}-y \\
0, & \text { otherwise. }
\end{array}\right.
\end{aligned}
$$

Taking above variations and (8) into account and applying bivariate integration by parts method to last inequality, we have

$$
\begin{aligned}
I_{1121}^{*} \leq & -\varepsilon \int_{x_{0}-x}^{x_{0}-x+\delta} \int_{y_{0}-y-\delta}^{y_{0}-y}\left[E_{1}(t, s)+E_{2}(t)+E_{3}(s)+K_{\lambda}\left(\sqrt{\left(x_{0}-x+\delta\right)^{2}+\left(y_{0}-\delta-y\right)^{2}}\right)\right] \\
& \times\left\{\left(t-x_{0}+x\right)^{\alpha+1}\right\}_{t}^{\prime}\left\{\left(y_{0}-s-y\right)^{\beta+1}\right\}_{s}^{\prime} d t d s \\
= & \varepsilon^{p}\left(i_{1}+i_{2}+i_{3}+i_{4}\right) .
\end{aligned}
$$

Using Remark 1 and condition (d) of class $A_{\varphi}$, we get

$$
\begin{aligned}
i_{1}+i_{2}+i_{3}+i_{4}= & -\int_{x_{0}-x}^{x_{0}-x+\delta} \int_{y_{0}-y-\delta}^{0} K_{\lambda}\left(\sqrt{t^{2}+s^{2}}\right)\left\{\left(t-x_{0}+x\right)^{\alpha+1}\right\}_{t}^{\prime} \\
& \times\left\{\left(y_{0}-s-y\right)^{\beta+1}\right\}_{s}^{\prime} d t d s
\end{aligned}
$$




$$
\begin{aligned}
& +\int_{x_{0}-x}^{x_{0}-x+\delta} \int_{0}^{y_{0}-y}\left(K_{\lambda}\left(\sqrt{t^{2}+s^{2}}\right)-2 K_{\lambda}(|t|)\right)\left\{\left(t-x_{0}+x\right)^{\alpha+1}\right\}_{t}^{\prime} \\
& \times\left\{\left(y_{0}-s-y\right)^{\beta+1}\right\}_{s}^{\prime} d t d s .
\end{aligned}
$$

Hence the following inequality holds for $I_{1121}^{*}$ :

$$
\begin{aligned}
I_{1121}^{*} \leq & \varepsilon^{p} \int_{x_{0}}^{x_{0}+\delta} \int_{y_{0}-\delta}^{y_{0}} K_{\lambda}\left(\sqrt{(t-x)^{2}+(s-y)^{2}}\right)\left|\left\{\left(t-x_{0}\right)^{\alpha+1}\right\}_{t}^{\prime}\right|\left|\left\{\left(y_{0}-s\right)^{\beta+1}\right\}_{s}^{\prime}\right| d t d s \\
& +2 \varepsilon^{p}\left|y_{0}-y\right|^{\beta+1} \int_{x_{0}}^{x_{0}+\delta} K_{\lambda}(|t-x|)\left|\left\{\left|x_{0}-t\right|^{\alpha-1}\right\}_{t}^{\prime}\right| d t .
\end{aligned}
$$

Analogous computations for $I_{1122}^{*}, I_{1123}^{*}$, and $I_{1124}^{*}$ yields

$$
\begin{aligned}
I_{1122}^{*} \leq & \varepsilon^{p} \int_{x_{0}-\delta}^{x_{0}} \int_{y_{0}-\delta}^{y_{0}} K_{\lambda}\left(\sqrt{(t-x)^{2}+(s-y)^{2}}\right)\left|\left\{\left(x_{0}-t\right)^{\alpha+1}\right\}_{t}^{\prime}\right|\left|\left\{\left(y_{0}-s\right)^{\beta+1}\right\}_{s}^{\prime}\right| d t d s \\
& +2 \varepsilon^{p}\left|y_{0}-y\right|^{\beta+1} \int_{x_{0}-\delta}^{x_{0}} K_{\lambda}(|t-x|)\left|\left\{\left|x_{0}-t\right|^{\alpha-1}\right\}_{t}^{\prime}\right| d t \\
& +2 \varepsilon^{p}\left|x_{0}-x\right|^{\alpha+1} \int_{y_{0}-\delta}^{y_{0}} K_{\lambda}(|s-y|)\left|\left\{\left|y_{0}-s\right|^{\beta+1}\right\}_{s}^{\prime}\right| d s \\
& +4 \varepsilon^{p} K_{\lambda}(0)\left|x_{0}-x\right|^{\alpha+1}\left|y_{0}-y\right|^{\beta+1} ; \\
I_{1123}^{*} \leq & \varepsilon^{p} \int_{x_{0}-\delta}^{x_{0}} \int_{y_{0}}^{y_{0}+\delta} K_{\lambda}\left(\sqrt{(t-x)^{2}+(s-y)^{2}}\right)\left|\left\{\left(x_{0}-t\right)^{\alpha+1}\right\}_{t}^{\prime}\right|\left|\left\{\left(s-y_{0}\right)^{\beta+1}\right\}_{s}^{\prime}\right| d t d s \\
& +2 \varepsilon^{p}\left|x_{0}-x\right|^{\alpha+1} \int_{y_{0}}^{y_{0}+\delta} K_{\lambda}(|s-y|)\left|\left\{\left|y_{0}-s\right|^{\beta+1}\right\}_{s}^{\prime}\right| d s ;
\end{aligned}
$$


$I_{1124}^{*} \leq \varepsilon^{p} \int_{x_{0}}^{x_{0}+\delta} \int_{y_{0}}^{y_{0}+\delta} K_{\lambda}\left(\sqrt{(t-x)^{2}+(s-y)^{2}}\right)\left|\left\{\left(t-x_{0}\right)^{\alpha+1}\right\}_{t}^{\prime}\right|\left|\left\{\left(s-y_{0}\right)^{\beta+1}\right\}_{s}^{\prime}\right| d t d s$.

Hence the following inequality is obtained for $I_{112}$ :

$$
\begin{aligned}
I_{112} \leq \varepsilon^{p} & \sup _{(t, s) \in B_{\delta}} \varphi(t, s)\left\{\int_{x_{0}-\delta}^{x_{0}+\delta} \int_{y_{0}-\delta}^{y_{0}+\delta} K_{\lambda}\left(\sqrt{(t-x)^{2}+(s-y)^{2}}\right)\right. \\
& \times\left|\left\{\left|x_{0}-t\right|^{\alpha+1}\right\}_{t}^{\prime}\right|\left|\left\{\left|y_{0}-s\right|^{\beta+1}\right\}_{s}^{\prime}\right| d t d s \\
& +2\left|y_{0}-y\right|^{\beta+1} \int_{x_{0}-\delta}^{x_{0}+\delta} K_{\lambda}(|t-x|)\left|\left\{\left|x_{0}-t\right|^{\alpha+1}\right\}_{t}^{\prime}\right|^{\prime} d t \\
& +2\left|x_{0}-x\right|^{\alpha+1} \int_{y_{0}-\delta}^{y_{0}+\delta} K_{\lambda}(|s-y|)\left|\left\{\left|y_{0}-s\right|^{\beta+1}\right\}_{s}^{\prime}\right| d s \\
& \left.+4 K_{\lambda}(0)\left|x_{0}-x\right|^{\alpha+1}\left|y_{0}-y\right|^{\beta+1}\right\} .
\end{aligned}
$$

The remaining part of the proof is obvious by the hyphothesis (7). The case $0<x-x_{0}<\delta / 2$ and $0<y-y_{0}<\delta / 2$ for $0<\delta<\infty$ can be proved in similar way. Besides, the proof of the assertion for $p=1$ is similar to the above one and thus is omitted. Therefore, the proof is completed.

\section{Rate of Convergence}

In this section, we give a theorem about the rate of point-wise convergence.

Theorem 2. Assume that the hypothesis of Theorem 1 is satisfied. Let

$$
\begin{aligned}
\Delta(\lambda, \delta, x, y)= & \sup _{(t, s) \in B_{\delta}} \varphi(t, s)\left\{\int_{x_{0}-\delta}^{x_{0}+\delta} \int_{y_{0}-\delta}^{y_{0}+\delta} K_{\lambda}\left(\sqrt{(t-x)^{2}+(s-y)^{2}}\right)\right. \\
& \times\left|\left\{\left|x_{0}-t\right|^{\alpha+1}\right\}_{t}^{\prime}\right|\left|\left\{\left|y_{0}-s\right|^{\beta+1}\right\}_{s}^{\prime}\right| d t d s
\end{aligned}
$$




$$
\begin{aligned}
& +2\left|y_{0}-y\right|^{\beta+1} \int_{x_{0}-\delta}^{x_{0}+\delta} K_{\lambda}(|t-x|)\left|\left\{\left|x_{0}-t\right|^{\alpha+1}\right\}_{t}^{\prime}\right| d t \\
& +2\left|x_{0}-x\right|^{\alpha+1} \int_{y_{0}-\delta}^{y_{0}+\delta} K_{\lambda}(|s-y|)\left|\left\{\left|y_{0}-s\right|^{\beta+1}\right\}_{s}^{\prime}\right| d s \\
& \left.+4 K_{\lambda}(0)\left|x_{0}-x\right|^{\alpha+1}\left|y_{0}-y\right|^{\beta+1}\right\},
\end{aligned}
$$

for $\delta>0$ and the following assumptions are satisfied:

(i) $\Delta(\lambda, \delta, x, y) \rightarrow 0$ as $(x, y, \lambda) \rightarrow\left(x_{0}, y_{0}, \lambda_{0}\right)$ for some $\delta>0$.

(ii) Letting $(x, y, \lambda) \rightarrow\left(x_{0}, y_{0}, \lambda_{0}\right)$, we have

$$
\left|\frac{1}{\varphi\left(x_{0}, y_{0}\right)} \iint_{\mathbb{R}^{2}} \varphi(t, s) K_{\lambda}\left(\sqrt{(t-x)^{2}+(s-y)^{2}}\right) d t d s-1\right|=\mathrm{o}(\Delta(\lambda, \delta, x, y)) .
$$

(iii) For every $\xi>0$,

$$
\sup _{\xi \leq \sqrt{\left(t^{2}+s^{2}\right)}}\left[\varphi(t, s) K_{\lambda}\left(\sqrt{(t-x)^{2}+(s-y)^{2}}\right)\right]=\mathrm{o}(\Delta(\lambda, \delta, x, y))
$$

as $(x, y, \lambda) \rightarrow\left(x_{0}, y_{0}, \lambda_{0}\right)$.

(iv) $\forall \xi>0$,

$$
\iint_{\xi \leq \sqrt{\left(t^{2}+s^{2}\right)}} \varphi(t, s) K_{\lambda}\left(\sqrt{(t-x)^{2}+(s-y)^{2}}\right) d t d s=\mathrm{o}(\Delta(\lambda, \delta, x, y))
$$

as $(x, y, \lambda) \rightarrow\left(x_{0}, y_{0}, \lambda_{0}\right)$. 
Then at each common p-generalized Lebesgue point of $f \in L_{p, \varphi}\left(\mathbb{R}^{2}\right)$

and $\varphi$, we have as $(x, y, \lambda) \rightarrow\left(x_{0}, y_{0}, \lambda_{0}\right)$

$$
\left|L_{\lambda}(f ; x, y)-f\left(x_{0}, y_{0}\right)\right|^{p}=\mathrm{o}(\Delta(\lambda, \delta, x, y))
$$

Proof. The assertion is obvious by the hypothesis of Theorem 1 .

Example 1. Let $\Lambda=(0, \infty), \lambda_{0}=0, \varphi(t, s)=(1+|t|)(1+|s|)$ and

$$
H_{\lambda}(t, s)=\frac{1}{4 \pi \lambda} e^{\frac{-\left(t^{2}+s^{2}\right)}{4 \lambda}}
$$

To verify that $H_{\lambda}(t, s)$ satisfies the hypotheses of Theorems 1 and 2 , see [12]. Also, the given weight function satisfies the hypotheses of Theorems 1 and 2 .

\section{Acknowledgements}

The authors thank the anonymous referee for his/her valuable suggestion and comments.

\section{References}

[1] C. Bardaro, On approximation properties for some classes of linear operators of convolution type, Atti Sem. Mat. Fis. Univ. Modena 33(2) (1984), 329-356.

[2] C. Bardaro and C. Gori Cocchieri, On the degree of approximation for a class of singular integrals, Rend. Mat. (7) 4(4) (1984), 481-490 (Italian).

[3] S. Bochner and K. Chandrasekharan, Fourier Transforms, Annals of Mathematics Studies, no. 19, Princeton University Press, Princeton, N. J. Oxford University Press, London, ix+219 (1949).

[4] A. D. Gadjiev, On the order of convergence of singular integrals which depend on two parameters, Special Problems of Functional Analysis and their Appl. to the Theory of Diff. Equation and the Theory of Func., Izdat. Akad. Nauk Azerbaĭdažan. SSR. (1968), 40-44.

[5] S. R. Ghorpade and B. V. Limaye, A Course in Multivariable Calculus and Analysis, Springer, New York, 2010.

[6] H. Karsli and E. Ibikli, On convergence of convolution type singular integral operators depending on two parameters, Fasc. Math. (38) (2007), 25-39. 
[7] R. G. Mamedov, On the order of convergence of $m$-singular integrals at generalized Lebesgue points and in the space $L_{p}(-\infty, \infty)$, Izv. Akad. Nauk. SSSR Ser. Mat. 27(2) (1963), 287-304.

[8] B. Rydzewska, Approximation des fonctions de deux variables par des intégrales singulières doubles, Fasc. Math. (8) (1974), 35-45.

[9] B. Rydzewska, Approximation des fonctions par des intégrales singulières ordinaires, Fasc. Math. (7) (1973), 71-81.

[10] W. Rudin, Real and Complex Analysis, Mc-Graw Hill Book Co., London, 1987.

[11] S. Siudut, A theorem of Romanovski type for double singular integrals, Comment. Math. Prace Mat. 29 (1989), 277-289.

[12] S. Siudut, On the convergence of double singular integrals, Comment. Math. Prace Mat. 28(1) (1988), 143-146.

[13] E. M. Stein, Singular Integrals and Differentiability of Functions, Princeton Univ. Press, New Jersey, 1970.

[14] R. Taberski, On double integrals and Fourier series, Ann. Polon. Math. 15 (1964), 97-115.

[15] R. Taberski, On double singular integrals, Rocznicki Polskiego towarzystwa matematycznego, Seria I. Prace Matematyczne XIX (1976), 155-160.

[16] R. Taberski, Singular integrals depending on two parameters, Rocznicki Polskiego towarzystwa matematycznego, Seria I. Prace Matematyczne VII (1962), 173-179.

[17] G. Uysal, M. M. Yilmaz and E. Ibikli, Approximation by radial type multidimensional singular integral operators, Palest. J. Math., to appear.

[18] G. Uysal, M. M. Yilmaz and E. Ibikli, A study on pointwise approximation by double singular integral operators, J. Inequal. Appl. 2015 (2015), 94.

[19] G. Uysal and M. M. Yilmaz, Some theorems on the approximation of non-integrable functions via singular integral operators, Proc. Jangjeon Math. Soc. 18(2) (2015), 241-251.

[20] M. M. Yilmaz, G. Uysal and E. Ibikli, A note on rate of convergence of double singular integral operators, Adv. Difference Equ. 2014 (2014), 287.

[21] M. M. Yilmaz, On convergence of singular integral operators depending on three parameters with radial kernels, Int. Journal of Math. Analysis 4(39) (2010), 1923-1928.

[22] M. M. Yilmaz, S. K. Serenbay and E. Ibikli, On singular integrals depending on three parameters, Appl. Math. Comput. 218(3) (2011), 1132-1135. 